Itinéraires Itinéraires

Littérature, textes, cultures

\title{
"Ceci est un récit » : prétentions et imaginaires narratifs en régime médiatique et marchand
}

\author{
Ambre Abid-Dalençon
}

\section{OpenEdition}

Journals

Édition électronique

URL : http://journals.openedition.org/itineraires/2647

DOI : $10.4000 /$ itineraires.2647

ISSN : 2427-920X

Éditeur

Pléiade

Référence électronique

Ambre Abid-Dalençon, « « Ceci est un récit » : prétentions et imaginaires narratifs en régime médiatique et marchand », Itinéraires [En ligne], 2015-1 | 2015, mis en ligne le 18 décembre 2015, consulté le 03 mai 2019. URL : http://journals.openedition.org/itineraires/2647 ; DOI : 10.4000/ itineraires. 2647

Ce document a été généré automatiquement le 3 mai 2019.

\section{(i)(3)}

Itinéraires est mis à disposition selon les termes de la licence Creative Commons Attribution - Pas d'Utilisation Commerciale - Pas de Modification 4.0 International. 


\title{
«Ceci est un récit » : prétentions et imaginaires narratifs en régime médiatique et marchand
}

\author{
Ambre Abid-Dalençon
}

\section{Introduction}

1 La journée d'étude à l'origine de cet article interrogeait un objet de recherche difficile à embrasser : les "récits de société ». De nombreuses formes considérées comme récits circulent en effet dans notre société mais semblent échapper constamment à l'analyste du fait de leur caractère complexe, diffus et protéiforme. La lecture des travaux sur ce qui serait des formes de récit - récits autobiographiques, récits d'enquête, récits de presse, mais aussi docufictions, publicités, serious games, etc.- peut déconcerter tant ils témoignent des différentes incarnations possibles de l'objet. Les formes textuelles ou plus largement sémiotiques que l'on attribue tour à tour au récit sont donc extrêmement variées et contrastées. De plus, aux divers horizons disciplinaires des chercheurs répondent autant de définitions, d'enjeux de recherche et d'approches méthodologiques.

Cette vision transformant la société en un "tout récit » fait écho pour le chercheur à l'extension du domaine de la narratologie qui se serait amorcée dès les années 1980. On parle alors de narratologie post-classique. On s'arrêtera dans un premier temps sur ce paysage théorique dans lequel semble s'inscrire l'expression " récits de société ».

3 L'étendue du champ ainsi dessinée, il faudra opter pour un parti pris afin d'aborder la variété des possibles de l'objet. Cet article propose une approche communicationnelle de ces récits. On questionnera leur circulation et les prétentions communicationnelles (Jeanneret 2008) qui entourent certaines mises en récit sur la scène médiatique et marchande. Pour finir, on interrogera les enjeux de pouvoir que sous-tend le projet narratif chez certains professionnels du marketing et de la communication.

4 En termes de corpus, on se concentrera sur des formes publicitaires narrativisées, déployées dans le cadre de tactiques de storytelling ${ }^{1}$ et de contenu de marque ${ }^{2}$, qui 
participent à leur manière du paysage médiatique. Ces deux notions amèneront à penser certains « récits de société » sous le prisme de pratiques professionnelles en marketing et communication.

5 Cet article s'inscrit dans le cadre d'une recherche doctorale en cours. Il propose donc d'apporter des pistes de réflexion plutôt que des réponses, à travers des questions que nous nous posons dans l'état actuel des recherches. Une contribution que nous espérons féconde, malgré son caractère inachevé, dans le cadre de ce numéro.

\section{" Récits de société » : un objet extensif et sous tension}

\section{Extension de l'objet « récit »}

Parler de récit suppose d'analyser cette modalité d'énonciation bien précise qu'est le narratif, objet de la narratologie. Cette dernière connaît une extension de son domaine d'étude depuis plusieurs dizaines d'années :

Ne désignant plus tout juste un sous-ensemble de la théorie littéraire structuraliste, narratologie peut maintenant s'employer pour désigner toute approche raisonnée de l'étude du discours narrativement organisé, qu'il soit littéraire, historiographique, conversationnel, filmique ou autre. (Herman 1999: 27, dans Prince 2014)

Dans l'article « Narratologie classique et narratologie post-classique » dont est extraite cette citation, Gerald Prince propose un état des lieux clair sur la question. Comme pour de nombreux auteurs, la narratologie post-classique reprend selon lui les questions fondamentales de la narratologie classique sur le récit ainsi que certaines de ses bases théoriques pour les re-contextualiser et en éprouver les limites. Par exemple, le récit est interrogé dans sa fonction et non plus seulement dans son fonctionnement, il est appréhendé comme processus et pas uniquement comme résultat d'une production, une place plus importante est accordée au récepteur, etc. Plus simplement, il semblerait que l'étude des propriétés des textes narratifs ne soit plus centrale mais mise en perspective par l'étude des interactions entre le texte, ses contextes et ses réceptions.

La narratologie devient ainsi une catégorie de compréhension beaucoup plus « modeste » pour certains chercheurs, qui ont abandonné l'ambition de trouver un modèle unique, descriptif et explicatif des récits :

[...] s'il existe probablement une compétence discursive générale permettant à chacun de distinguer intuitivement - et très globalement - un récit d'un non-récit, les recherches en narratologie montrent qu'il n'y a pas, à l'heure actuelle, de définition univoque des concepts de narrativité et de récit. (Revaz 2009 : 70)

\section{Un déplacement de la question des « frontières du récit » : l'élasticité de la notion}

Cette extensivité du récit engage le croisement de multiples outils et méthodologies, mais pose par là même de nombreux problèmes épistémologiques. Ansgar Nünning met par exemple en garde contre l'usage inflationniste de la notion, la « force centrifuge de la narratologie » (Barry 1990, dans Nünning $2010: 22$ ) et tente de circonscrire son usage à venir : 
[...] on ferait mieux d'utiliser le terme « narratologie » avec plus de prudence et de réexaminer d'un œil critique la prolifération de narratologies toujours plus nombreuses. On ne saurait nier que la narratologie, tel un phénix, s'est récemment relevée de ses cendres pour devenir « un champ en évolution dynamique »; mais il semble douteux que toutes les « approches post-classiques » constituent vraiment de nouvelles branches ou de nouvelles versions de la narratologie. (Nünning 2010 : 32)

10 Afin de dépasser ce «tout ou rien narratif » (Revaz 2009), les métaphores ont proliféré chez les chercheurs pour désigner cette nouvelle narratologie contemporaine, faite de récits épars, hétérogènes et hybrides. Le récit n'est plus forcément appréhendable dans sa totalité mais prend la forme de la trace, de l'indice ou du fragment. Des approches théoriques plus souples sont proposées, invitant à déployer une nouvelle herméneutique pour faire face à ces "nouvelles" formes de récit. Françoise Revaz propose d'appréhender les récits anciens et actuels sur la base d'un continuum qui les positionnerait en fonction de leur « degré de narrativité », ce dernier étant défini selon des critères majeurs : chronologie, mise en intrigue, liens de causalité, etc. Jean-Michel Adam (2011: 25) rappelle que le suffixe «-ité » du terme «narrativité » met en évidence qu'il ne s'agit que d'une "potentialité attributive». Il montre que les textes ne peuvent appartenir que graduellement à tels ou tels classes ou genres de discours, selon ce qu'il appelle un " gradient de typicalité ». Philippe Marion va encore plus loin quand il propose de penser la narrativité dans sa virtualité, avec la notion de « ferment narratif »:

[...] on peut constater le caractère narratif de tel objet observé (par exemple, un film de fiction), mais on peut aussi saisir du ferment narratif dans tel autre objet (par exemple, une photographie qui suggère un récit qu'elle ne contient pas) [...]. La narrativité serait non seulement un résultat mais elle contiendrait aussi une dimension promissive, celle d'un potentiel ou d'un développement virtuel suggérés par la présence simultanée de certains indices. (Marion 1997 : 84)

11 Il ne s'agit donc plus de définir les «frontières du récit " (Genette 1966), mais d'analyser ses effets de dispersion. L'élasticité et la plasticité de la notion sont reines. Il semble cependant que ces images invoquées traduisent parfois en creux la difficulté à cerner cet objet.

\section{« Récits de société » : quelle approche critique?}

12 Si la narrativité peut aujourd'hui se penser avec une telle amplitude, on peut se demander ce que définit encore la catégorie de récit. Naviguant entre deux eaux, si on ne peut certes plus appliquer certaines catégories d'analyse textuelle - parce que trop rigides - à certains objets, on reste parfois insatisfait face à des catégories variables, qui bien que précieuses sur le plan d'une réflexion épistémologique, ne peuvent suffire à constituer un cadre d'analyse. C'est en ce sens que le récit, pris dans son extensivité, est un objet fondamentalement interdisciplinaire : les analyses textuelles, quand elles sont possibles, doivent se conjuguer avec d'autres approches. Le curseur est changeant et l'analyse n'est possible qu'à l'intersection de ces divers regards.

Tout dépend par ailleurs de la posture initiale face aux objets «récits»: quelle compréhension avons-nous de la préposition « de » dans une formule telle que « récits de société » ? En transposant à notre sujet les questions que pose Yves Jeanneret à propos de l'expression "technologies de l'information " (Jeanneret 2007: 58), dans l'expression "récits de société » les récits sont-ils "fabriqués avec" la société ou "fabricants de" société ? Habitent-ils la société sans forcément se poser comme récits? En ce cas, nous 
pouvons les en extraire pour nos analyses et les transformer en révélateurs discursifs de nos réalités sociales. Ou bien, ces derniers se proposent-ils manifestement de raconter la société et c'est en tant que tels que nous les appréhendons?

Dans une démarche communicationnelle, on se concentrera sur cette deuxième proposition. Après avoir présenté très succinctement certaines réflexions théoriques sur le récit qui témoignent d'une extension de la notion et de certaines difficultés concomitantes à celle-ci, la démarche de cet article consiste non pas à dire quand il y a récit ou non, mais à réfléchir aux conditions qui permettent à certains récits d'advenir dans l'espace public et médiatique en tant que récits, et à interroger cette promotion de l'objet. Cette approche mobilise les notions de la trivialité et de la prétention communicationnelle développées par Yves Jeanneret.

\section{Pour une approche communicationnelle des « récits de société »}

\section{Trivialité de la forme narrative}

La notion de trivialité permet de discuter en termes communicationnels les conditions d'existence, de dénomination et de circulation des récits. Sans aucune connotation péjorative, le terme «trivialité » est pris par l'auteur dans son étymologie latine, au sens de "carrefour ». Yves Jeanneret définit la trivialité dès les premières lignes de son ouvrage comme le phénomène qui fait que «[...] les objets et les représentations ne restent pas fermés en eux-mêmes mais circulent et passent entre les mains et les esprits des hommes " (Jeanneret 2008: 14). La trivialité est donc le processus par lequel les représentations, savoirs, valeurs ou encore expériences esthétiques en circulant se transforment, se pérennisent, donnent sens. Jeanneret va plus loin: c'est par ces échanges et appropriations successives qu'un objet acquiert de la valeur. Il faut donc bien entendre la trivialité de ce qu'il nomme les «êtres culturels " comme une pratique fondamentalement " productive ». Ainsi le storytelling actualise, en se la réappropriant, la modalité narrative à chaque campagne - médiatique, politique, promotionnelle, etc. Que ce soit sous forme de macro ou de micro-récits, cette circularité amène la création en continu de nouveaux espaces narratifs. C'est en cela que l'on peut rattacher la trivialité de ces récits à une pratique productive.

Toujours selon Yves Jeanneret, chaque "être culturel » présente une historicité et un mode d'existence sociale; il est donc important dans une telle perspective de s'attacher tant aux objets qu'aux pratiques, procédures et «[...] phénomènes de qualification, de sélection, de hiérarchisation des œuvres et des idées » (Ibid : 59). On pourrait donc penser les pratiques et processus sociaux liés aux récits afin d'appréhender les représentations, imaginaires, savoirs ou valeurs qui se cristallisent autour de cette forme quand on fait appel à elle dans un contexte médiatique, et plus précisément ici marchand.

Étudier les formes de médiation contemporaines du récit, c'est étudier l'idée du récit qui circule, pour que ce dernier soit lu, reconnu, compris comme « récit ». Mais c'est analyser aussi les procédés de valorisation qui ont cours à travers l'usage de cette forme. En empruntant la forme narrative dans leurs stratégies communicationnelles, les professionnels de la communication nous livrent un point de vue sur « ce qui fait récit ", une certaine idée de l'écriture narrative et par là même de la communication. 


\section{Prétention narrative et prétention communicationnelle} plusieurs âges qui formeraient "un arc herméneutique entre Balzac et Barthes» (Boutaud et Veron 2007: 51). Chacun de ces âges est commandé par un paradigme publicitaire, défini en fonction de certains traits dominants : "expression » (importance du littéraire dans la réclame et l'annonce), "création" (âge d'or des peintres et affichistes), "persuasion» (influence des thèses psychologistes et motivationnistes) et «signification» (Barthes et la sémiologie). Selon ces auteurs, ces divers âges ont tous participé d'une façon ou d'une autre à la construction de « mythologies publicitaires ».

19

Cette hypothèse selon laquelle une représentation de la communication est mise en jeu au sein des dispositifs communicationnels est théorisée par Yves Jeanneret sous la notion de prétention communicationnelle. Analysant la manière dont s'instituent certaines pratiques et procédures professionnelles, Jeanneret définit la prétention communicationnelle comme «[...] le lien qui s'établit entre une conception de la communication et un projet d'intervenir sur elle » (Jeanneret $2014: 248$ ). Chaque paradigme publicitaire cité plus haut renvoie à une intention déployée en vue d'agir sur le processus communicationnel, soit à une manière différente de communiquer, laquelle s'accompagne de compétences variées. Comme le précise Jeanneret, la formulation d'une "prétention»-le sens du terme l'indique - n'engage en rien les pratiques elles-mêmes, ces dernières ne s'y conformant pas nécessairement. Mais cette dernière n'en informe pas moins sur une vision (faite de représentations, d'imaginaires, de croyances) et une visée (appuyée sur des procédures, des dispositifs, des méthodes) communicationnelles à une période donnée.

À la lumière de cette notion, on peut se demander dans quelle mesure le «tournant narratif ", tel qu'il peut se déployer dans les secteurs professionnels de la communication, est porteur de nouvelles prétentions communicationnelles. À étudier les discours des professionnels du marketing et de la publicité, il semble qu'il faille " faire du récit » pour (bien) communiquer aujourd'hui. Proposer $d u$ narratif est donc une forme de redéfinition et de réajustement de ce que doit être une bonne communication. L'usage de l'énonciation narrative dans les sphères professionnelles renvoie à une valorisation de cette forme circulante, apte à servir l'expression d'un modèle communicationnel.

\section{Monstration de l'appartenance narrative}

21 Face à la multitude des récits environnants, notre point de départ est la mise en récit explicite qui se donne à voir comme telle. C'est pourquoi nous préférons parler de mise en récit et non pas de récit. Les mises en récit produites par certains publicitaires présentent un condensé sémiotique pour le chercheur car ils « sur-signifient » leur filiation au récit. Cette saturation du signe est inhérente au fonctionnement du discours publicitaire qui, pour être lisible et visible, se doit de procéder par optimisation sémiotique :

Le discours publicitaire peut ainsi se définir par l'incidence formelle de ses conditions de production [...]. Le discours se trouve ainsi pris dans un système de densification qui aboutit le plus souvent à la recherche de formes à haute densité sémiotique et/ou créativement atypiques malgré la propension au stéréotype. (Berthelot-Guiet $2013: 275$ )

Pour reprendre les termes de Barthes, les storytelling des marques sont des récits «pleins » et "emphatiques $»^{3}$. Ces storytelling sont un exemple type de monstration de 
mises en récit car ils se mettent en scène en tant qu' «art de raconter ». Les marques de luxe sont à ce titre de grandes productrices de publicités narrativisées, comme en atteste L'Odyssée de Cartier ${ }^{4}$.

Ce film raconte la marque dans une logique de contenu de marque. Pour rappel, le contenu de marque désigne les opérations d'édition et de création de " contenus " par les marques (Bô et Guevel 2009). Ces « contenus " peuvent être de natures diverses (pratique, ludique, informative ou culturelle). Ils peuvent être scénarisés (dans le cadre d'une websérie ou d'un court-métrage comme ici) mais pas nécessairement. En ce sens, on peut lier storytelling et contenu de marque sans qu'ils ne se substituent l'un à l'autre. L'Odyssée de Cartier est un film institutionnel qui sert l'image et la notoriété de la marque, et qui répond à ces objectifs par une offre de «divertissement » de trois minutes trente dont l'objectif est de «faire connaître la culture et le patrimoine créatif de Cartier ${ }^{5}$ ». Afin de captiver le public, la logique du récit fonctionne à plein dans ce court-métrage : l'histoire débute à l'aube à Paris, rue de la Paix (la perspective du plan fait voir la colonne Vendôme). Dès les premières secondes, l'icône de la marque apparaît : une panthère toute sertie de pierres, placée dans une vitrine la mettant en scène. Le bijou est en effet placé sur un piédestal au centre d'un bâtiment en coupe à colonnes, surmonté d'une coupole ouverte permettant à la lumière de s'infiltrer. La statue de la panthère ainsi panthéonisée (l'échelle a changé au fur et à mesure des plans) prend soudain vie sous la lumière du jour. Sur le registre de l'épopée - exprimée par la course du félin et de tout le bestiaire environnant, ainsi que le rythme de la bande-son -, on suit le voyage de cette icône. Un voyage scandé par des lieux emblématiques de l'histoire de la marque représentés sous un jour particulièrement romanesque (la lancée d'une troïka dans un Saint-Pétersbourg enneigé, la confrontation au dragon transformé en Muraille de Chine, le palais d'un maharadjah en Inde construit sur le dos d'un éléphant), sans oublier bien sûr les motifs fantastiques (la statue qui prend vie, le dragon, un jardin composé de joyaux vivants). Le récit se clôt enfin par un retour sur la Place Vendôme, à la tombée de la nuit. L'animal rejoint sa maîtresse, parée d'un bracelet en forme de panthère, dont les pierres rappellent le bijou des premières secondes du film. Si toute cette narration sert un « univers de marque » représentant son histoire, ses créations et ses influences, L'Odyssée de Cartier révèle pour nous surtout une sur-sémiotisation d'éléments faisant récit (unité temporelle, enchaînement d'actions, construction d'un univers diégétique, registres de l'épopée, du fantastique et de l'onirique, procédés métonymiques et métaphoriques, épanadiplose narrative, etc.).

Que ce soit donc à travers des spots publicitaires ou des dispositifs plus complexes de contenu de marque, le storytelling sur-signifie son appartenance à la logique narrative, baptisant ces communications par une formule implicite: "ceci est un récit ${ }^{6}$ ». Un procédé d'affirmation identitaire que Karine Berthelot-Guiet analyse au regard des procédés autonymiques dans le discours publicitaire. L'autonymie - procédé par lequel le signe peut se désigner lui-même et définir les conditions de son emploi - est en effet utile pour la marque désireuse d'affirmer son identité (Berthelot-Guiet 2003) ${ }^{7}$. Le parallèle est ici intéressant pour aborder certaines formes narratives publicitaires, ces dernières s'affirmant et s'auto-légitimant comme telles, communiquant par-delà leur message premier que les objets ou informations sont bien en train et dignes d'être contés.

Mais pourquoi avoir recours à de telles stratégies? Quelles représentations du narratif, de la communication et plus globalement de la culture cela convoque-t-il ? Il s'agirait d'appréhender ces formes narratives dans leur projet et de comprendre ce qu'elles 
révèlent des imaginaires contemporains; une posture qui amène à considérer les objets médiatiques et marchands comme des lieux de fixation de certains imaginaires circulant dans la société. Ce qu'Eliseo Veron théorise par la notion de sémiosis sociale :

[...] tout acte de discours au sein de la communication médiatique met en jeu des éléments essentiels de la société et de la culture. Le message le plus « insignifiant » [...] active des composantes fondamentales de la sémiosis sociale [...]. Toute communication interpelle l'individu comme noyau d'appartenances. (Boutaud et Veron $2007: 19)$

Nous avons vu dans cette partie que le concept de trivialité d'Yves Jeanneret pouvait aider à rendre compte de la circulation des "êtres culturels" que sont les récits de société. Ces récits ont une vie sociale : ils émergent, disparaissent et réapparaissent en fonction des contextes. En tant que forme valorisée, la narration intéresse les sphères professionnelles de la communication qui se la réapproprient à leur tour. Dans la dernière partie de cet article, nous allons montrer que ces narrativités marchandes révèlent des intentions bien précises - quoique paradoxales parfois - de la part des professionnels.

\section{Imaginaires et ambiguiités du projet narratif}

\section{Imaginaires du pouvoir narratif}

On attribue communément un certain nombre de pouvoirs au récit. Le récit séduit de prime abord, tant par sa langue que par l'histoire déployée : le récit ne s'adresserait pas à la raison. Il capture ensuite : enfermé dans l'univers diégétique qui lui est conté, le public ne pourrait s'y soustraire. Dans un autre ordre d'idée, le récit est aussi ce qui organise et de fait donne sens au chaos des événements.

Certains de ces pouvoirs attribués au récit rejoignent ceux que l'on assigne, souvent à tort, aux discours communicationnels. Dans une pensée instrumentale du langage, où une action de l'émetteur produirait une réaction chez le récepteur, la communication - surtout si elle est médiatique car elle use d'outils à destination du plus grand nombre est parfois diabolisée. La méfiance - ou a contrario une certaine fascination - s'installe donc d'autant plus lorsque les pouvoirs attribués au récit semblent servir des stratégies communicationnelles. Cette fascination est particulièrement perceptible dans les discours de certains professionnels sur le storytelling, notamment dans les manuels censés former à ces techniques. En introduction de son ouvrage, voici les propos de Yaël Gabison, « experte en storytelling »:

Lorsque j'ai voulu comprendre comment je pouvais appliquer les techniques du storytelling, je n'ai trouvé aucune recette, aucune méthode concrète. Pourtant le storytelling est une merveilleuse technique. Très peu de gens le savent vraiment. Ils sont encore moins à la révéler. (Gabison 2012 : 15)

La magie de la technique est ici palpable, la professionnelle en communication vend une solution qui semble des plus efficaces et dont l'objectif est simple: séduire, marquer, captiver son auditoire. Dans une pensée instrumentale du langage, l'experte vante un différentiel de conscience ("très peu de gens le savent vraiment») qui lui confère clairement un pouvoir par l'adjuvant narratif.

En ce sens le « piège » est bien double ici, tel que l'analyse Louis Marin. Dans Le récit est un piège ou encore Le Portrait du roi, Louis Marin montre la stratégie discursive de l'historien ou du conteur qui usent du pouvoir du récit face au puissant. C'est ainsi que l'historien 
recommande au roi le discours historiographique des guerres - en tant que « discours du réel » et « désintéressé »-, sous l'argument que ce dernier forcera bien plus l'adhésion du peuple qu'un récit explicitement élogieux. Mais en opérant ainsi, ce dernier se rend indispensable, et la puissance du roi devient tributaire de celle du récit composé par l'historien compétent. Il est cependant trop tard. Avant même d'opérer sur le peuple, le récit d'une séduction à venir sur le peuple a séduit le roi : «[...] il conte au pouvoir la façon dont le pouvoir se raconte et, du même coup, le piégera à son propre piège par le plaisir que le pouvoir y prend» (Marin $1978: 11$ ). La « machinerie » du récit porte en elle un jeu, ce dernier ayant pour objet le désir de pouvoir de l'autre. Ce qui nous permet de faire le lien avec la notion de prétention communicationnelle déjà présentée :

En effet l'enjeu de la prétention est l'aptitude du professionnel de la communication à jouer avec le désir de représentation de ceux qui peuvent garantir son pouvoir et ses ressources et c'est ce qui explique l'importance du travail de représentation et de figuration de la communication. (Jeanneret $2014: 265$ )

L'argumentaire du storyteller est donc assez proche de celui de l'historien: le consommateur tomberait sous la coupe du pouvoir de la marque par le pouvoir du récit construit par le storyteller. Les notions d'imaginaire et de fantasme tiennent donc une place importante dans une telle réflexion, la logique instrumentale du langage témoignant d'un vieux fantasme de maitrise du langage chez les professionnels de la communication - étant entendu que c'est ainsi qu'ils promeuvent leurs compétences. D'où des réactions négatives à l'égard de ces techniques de storytelling, comme le montre le célèbre livre de Christian Salmon, Storytelling, la machine à fabriquer des histoires et à formater les esprits. L'auteur s'inquiète de la prolifération du récit devenu « instrument de contrôle » et démasque - sphère par sphère - «le nouvel ordre narratif ». À l'imaginaire du pouvoir et de la domination des signes répond ainsi souvent un autre imaginaire, celui d'un « décryptage » de ces mêmes signes.

Ancré depuis plusieurs années, le storytelling rend compte d'une certaine pensée du récit. Par ailleurs, la dimension communicationnelle de ces objets est au cœur des débats et interrogations. Derrière l'injonction du storyteller ou de ses détracteurs se cache un combat entre diverses prescriptions portant sur ce que doit être la communication. Les stratégies narratives qui nous préoccupent révèlent en effet une volonté de brouillage des frontières.

\section{Ambivalence du procédé narratif}

Nous avons vu aux paragraphes précédents qu'avec le contenu de marque la marque se donne pour mission de plonger le consommateur dans un univers éditorial. Ces tactiques sont commandées selon Valérie Patrin-Leclère, Caroline Marti de Montety et Karine Berthelot-Guiet (2014) par une logique de dépublicitarisation. Ce néologisme désigne la propension actuelle des annonceurs à se détacher des formes les plus classiques et reconnaissables de la publicité. Elles s'inscrivent dans un contexte de saturation médiatique, où la publicité classique se heurte à ses limites et où la consommation tendrait à se "culturaliser ». Plusieurs formes de culturalisation de la marque sont alors possibles par le contenu de marque : immixtion dans des formes médiatiques préexistantes (le placement de produit au cinéma), imitation de formes médiatiques instituées (le magazine de marque) ou encore production à part entière de formes culturelles (le musée) (Berthelot-Guiet 2013 : 294). Le recours au narratif dans ces dispositifs est alors récurrent ; le récit permet en effet une part d'ambivalence qui séduit les professionnels. 
34 L'univers de la marque ou bien son produit pourraient cependant sembler, dans une perspective publicitaire traditionnelle, très dilués - voire trop absents - de certaines narrations :

Prise dans des jeux rhétoriques - au sens large - de plus en plus complexes, la communication publicitaire prendra une dimension catachrétique de plus en plus criante (le décalé, le disruptif, l'oblique). Au point que la nature même du contrat avec les marques est remise en question, aujourd'hui, par des consommateurs parfois amusés, mais souvent désorientés par le discours publicitaire et ses coquecigrues. (Boutaud et Veron 2007 : 50)

Mais l'intérêt pour les professionnels réside justement dans ce mariage compliqué - et pourtant pas si neuf au regard de l'histoire publicitaire -, entre logique marchande et formes narratives. Ce mariage brouille les frontières et donne lieu à une nouvelle herméneutique.

\section{Esthétique du trouble}

Les tactiques de contenu de marque ayant recours au récit mêlent différentes logiques. Leur identification ainsi que leur délimitation - où se termine le divertissement? où commence le marchand? - sont compliquées voire impossibles. Cela suppose que le récepteur se place dans une posture de réception bien particulière, acceptant une confusion entre des genres et des logiques discursives. Soit un état de flottement et d'incertitude constant face à ces discours, qui confère à la fameuse "coopération interprétative » une latitude nouvelle. Il n'est pas sûr que nous ayons toujours en main les clés de la « situation de récit » dont parlait Barthes :

[...] tout récit est tributaire d'une "situation de récit », ensemble des protocoles selon lesquels le récit est consommé [...] si familier, si négligent que soit aujourd'hui le fait d'ouvrir un roman, un journal ou un poste de télévision, rien ne peut empêcher que cet acte modeste n'installe en nous, d'un seul coup et dans son entier, le code narratif dont nous allons avoir besoin. (Barthes $1966: 22$ )

Par exemple, l'essor des « nouvelles de marque » est à ce titre éclairant. En février 2010, Sofitel a lancé Les Escales littéraires, une opération qui continue aujourd'hui. Chaque mois, un écrivain est invité à passer un séjour dans un hôtel de son choix et à écrire une nouvelle autour de cette expérience : certains citent frontalement la marque, d'autres insèrent une à plusieurs descriptions élogieuses de l'hôtel d'accueil (situation paradisiaque, luxe du décor, amabilité du personnel, etc.), d'autres - certes plus rares sont très discrets et n'y font quasiment pas référence. Reste que ces glissements, qui font passer incidemment du statut de lecteur à celui de potentiel consommateur, invitent à entrer dans un exercice de lecture bien particulier. La densification recherchée dans le processus communicationnel par le recours au narratif amène alors une nouvelle poétique et esthétique du trouble, de l'opacité.

\section{Conclusion}

Nous avons proposé dans le cadre de cet article une approche communicationnelle du récit, devenu mise en récit, à la lumière d'un corpus médiatico-marchand. Nous avons tenté d'éclairer les conditions d'existence et de circulation de ces mises en récit dans les pratiques professionnelles du marketing et de la communication. Dans une telle optique, la question est de comprendre pourquoi certains acteurs souhaitent emprunter ces 
formes narratives, ce que cela signifie en termes d'imaginaires et d'enjeux de pouvoir. Quel que soit le lieu d'où on se place pour observer ces « récits de société », il importe en effet d'interroger le pouvoir que l'on souhaite leur prêter.

\section{BIBLIOGRAPHIE}

Adam, Jean-Michel, 2011, Genres de récits : narrativité et généricité des textes, Paris, L'Harmattan.

Barry, Jackson G., 1990, « Narratology's Centrifugal Force: A Literary Perspective on the Extensions of Narrative Theory ", Poetics Today, n 11 (2), p. 727-753.

Barthes, Roland, 1964, « Rhétorique de l'image », Communications, nº 4, p. 40-51.

-, 1966, «Introduction à l'analyse structurale des récits ", Communications, nº 8, p. 1-27.

Berthelot-Guiet, Karine, 2003, " "Ceci est une marque" : stratégies métalinguistiques dans le discours publicitaire ", Communication \& langages, $\mathrm{n}^{\circ} 136$, p. 58-71.

Berthelot-Guiet, Karine, 2013, Paroles de pub. La vie triviale de la publicité, Paris, Éditions Non Standard.

Bô, Daniel et Guével, Matthieu, 2009, Brand Content : comment les marques se transforment en médias, Paris, Dunod.

Boutaud, Jean-Jacques et Veron, Eliseo, 2007, Sémiotique ouverte : itinéraires sémiotiques en communication, Paris, Lavoisier.

Gabison, Yaël, 2012, Boostez vos présentations avec le storytelling, Paris, Eyrolles.

Genette, Gérard, 1966, « Frontières du récit », Communications, nº 8, p. 152-163.

Herman, David (dir.), 1999, Narratologies: New Perspectives on Narrative Analysis, Columbus, Ohio State University Press.

Jeanneret, Yves, 2007, Y a-t-il (vraiment) des technologies de l'information?, Villeneuve d'Ascq, Presses Universitaires du Septentrion.

-, 2008, Penser la trivialité, vol. 1 : La vie triviale des êtres culturels, Paris, Lavoisier.

-, 2014, Critique de la trivialité. Les médiations de la communication, enjeu de pouvoir, Paris, Éditions Non Standard.

Marin, Louis, 1978, Le récit est un piège, Paris, Minuit.

Marion, Philippe, 1997, « Narratologie médiatique et médiagénie des récits », Recherches en communication, $\mathrm{n}^{\circ} 7, \mathrm{p} .61-88$.

Nünning, Ansgar, 2010, « Narratologie ou narratologies? Un état des lieux des développements récents : propositions pour de futurs usages du terme », dans J. Pier et F. Berthelot (dir.), Narratologies contemporaines: approches nouvelles pour la théorie et l'analyse du récit, Paris, Éditions des archives contemporaines, p. 15-44.

Patrin-Leclère, Valérie, Marti de Montety, Caroline et Berthelot-Guiet, Karine, 2014, La Fin de la publicité ? Tours et contours de la dépublicitarisation, Lormont, Le Bord de l'Eau. 
Prince, Gerald, 2014, « Narratologie classique et narratologie post-classique », Vox Poetica, http:// www.vox-poetica.org/t/articles/prince.html.

Revaz, Françoise, 2009, Introduction à la narratologie. Action et narration, Bruxelles, De Boeck.

Salmon, Christian, 2007, Storytelling, la machine à fabriquer des histoires et à formater les esprits, Paris, La Découverte.

\section{NOTES}

1. Le "storytelling» renvoie aux techniques narratives qui se sont développées depuis les années 2000 dans les sphères tant politiques, journalistiques que publicitaires.

2. Le « contenu de marque » désigne l'opération de création et/ou d'édition de « contenus » par les marques. Nous y reviendrons plus loin.

3. «[...] si l'image contient des signes, on est donc certain qu'en publicité ces signes sont pleins, formés en vue de la meilleure lecture: l'image publicitaire est franche, ou du moins emphatique » (Barthes $1964:$ 40).

4. Disponible à l'adresse: http://www.cartier.fr/la-maison/styles-et-histoires/lodyssee-decartier (consulté le 10 mai 2015).

5. «Cartier, l'Odyssée fantastique", Stratégies.fr, disponible à l'adresse: http:// www.strategies.fr/grands-prix/246/grand-prix-strategies-amaury-medias-du-luxe-0/lelaureat/194954W/2-15-2581/cartier-l-odyssee-fantastique.html (consulté le 10 mai 2015).

6. Cela est d'autant plus perceptible lorsqu'il s'agit de «livres de marque » ou de nouvelles commandées à des écrivains par des marques : le format livre (qu'il soit matérialisé en format papier ou numérique) étant l'écrin par essence de la narrativité.

7. Le titre de notre article fait écho à celui-ci.

\section{RÉSUMÉS}

Cet article propose de réfléchir sous un angle communicationnel aux conditions de circulation et de valorisation de la forme narrative sur la scène médiatique et marchande, à travers l'exemple du storytelling et du contenu de marque. Face à ce qui est présenté par de nombreux auteurs comme une « extension du domaine narratif », on se concentrera sur des mises en récit explicites, qui se revendiquent comme telles. Ces formes «emphatiques" de mises en récit révèlent certains imaginaires autour de l'objet « récits de société ».

This paper studies the circulation and development conditions of narrative forms with a communicational approach on the media and advertising scenes. Considering the expansion and variety of narratives, the analysis will focus on some explicit and "emphatic" narrative forms of storytelling and brand content. These examples might reveal some representations around this object. 
INDEX

Mots-clés : récit, narrativité, prétention communicationnelle, imaginaire, storytelling, contenu de marque

Keywords : narrative, narration, storytelling, brand content, communicational approach

\section{AUTEUR}

AMBRE ABID-DALENÇON

Université Paris-Sorbonne, GRIPIC 